\title{
Regeneration der Kiefer bei Reptilien und Amphibien.
}

\author{
Von \\ Isaak Werber. \\ (Aus der Biologischen Versuchsanstalt in Wien.) \\ Mit Tafel I und II. \\ Eingegangen am 24. Mai 1906. \\ Inhaltsübersicht.

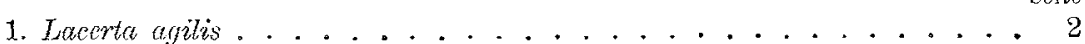

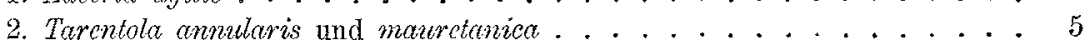 \\ 3. Triton eristatus und alpestris. . . . . . . . . . . . . . . 6 \\ 4. Rana csertlenta ..................... . 7 \\ 5. Rana temporaria . . . . . . . . . . . . . . 7 \\ 6. Hyla arborea . . . . . . . . . . . . . . . 8 \\ 7. Versuchsprotokolle . . . . . . . . . . . . . . . . 9 \\ 8. Erklärung der Abbildungen . . . . . . . . . . . . 13
}

In einer früheren Mitteilung ${ }^{1}$ ) habe ich iber die von mir experimentell ermittelte Tatsache der Regeneration der Kiefer bei der Eidechse Lacerta agilis berichtet. Die Ansichten von Lessona, WEISMann und Bordage, daß die Regenerationsfähigkeit eines gewissen Körperteiles oder Gliedes bei einem gewissen Tiere von seiner funktionellen Wichtigkeit und von der Verlustmöglichkeit abhängt, wie auch meine Beobachtungen bezüglich der Schnabelregeneration bei weiblichen Individuen vom Haushuhn gaben die unmittelbare Anregung zur Anstellung dieses Versuchs. Trotzdem bereits zahlreiche Versuche und Beobachtungen es klar dargetan haben, daß die Deutung der Regeneration im Sinne der genannten Autoren unhaltbar ist, sind noch immer manche Forscher bestrebt, deren Erklärungsweise der

1) Archiy f. Entw.-Mech. 1905. Bd. XIX. Heft 2. 
Regenerationserscheinungen im Tierreiche eifrig za verfechten $\left.{ }^{1}\right\}$ und zu begründen. WeIsmanN selbst erklärt die von KenNex bekannt gewordene Tatsache der Regeneration des Schnabels beim Storch unter Zuhilfenahme einer selectionstheoretischen Ableitung, als Anpassungserscheinung an die Verlustmöglichkeit des Schnabels beim männlichen Storche. Aus den Ansichten Wersmanns geht hervor, daß die Regeneration des Schnabels beim männlichen Storche als der einzige oder vielleicht als einer der wenigen Widersprïche gegen die adaptative Natur der Regeneration anzusehen war, der nun durch seine Erklärungsweise gänzlich aufgehoben werde. Durch die Bekanntgabe der Tatsache der Regeneration bei Individuen weiblichen Geschlechts vom Haushuhn und bei Individuen beiderlei Geschlechter von Lacerta agilis habe ich das Gegenteil nachznweisen gesucht. Da man vielleicht eine größere Verlustmöglichkeit der Kiefer bei der Lacerta agilis annehmen könnte (wofiir allerdings keinerlei Anbaltspunkte vorliegen), habe ich die Regenerationsfähigkeit der Kiefer noch bei andern Reptilien und auch bei Amphibien überprüft. Unter diesen war es von den Fröschen bislang nicht bekannt, daß sie als metamorphosierte Tiere einen ganzen Körperteil zu regenerieren vermögen. 'Tch will nun in aller Kirre noch einmal anf die Versuche an der Eidechse zurïekkommen, um dann über ähnliche Resultate bei andern Reptilien und Amphibien berichten zu können.

\section{Lacerta agilis $L$.}

Ich amputierte bei diesen Tieren den Kieferteil bis hart an die Grenze der Nasenlöcher (Intermaxillare) und einen entsprechend großen Teil am Unterkiefer (Symphysiale). Zum Versuche wurden Tiere männlichen und weiblichen Geschlechts versehiedenen Alters verwendet. Die Operation verursachte eine verhältnismäßig sehr geringe Blutung. Der WundverschluR erfolgte sehr raseb. Nach dem Abstoßen des Wundschorfs sah man die Wundränder mit Epithel überkleidet. Die winkelartige Wundlïcke am Oberkiefer füllte sich immer mehr bis sie gänzlich verschwand; hierauf wölbte sich die bereits geschlossene Wundfäche (siehe Fig. 2) und der Kiefer nahm

1) In einem populärwissenschaftlichen Werke, betitelt $>$ Der Darwinismus und die Probleme des Lebens von Dr. KonRaD GünTher (Freiburg i. Br., Fr. E. Fehsenfeld) werden die Wrismannschen Ansichten über die Regeneration und ihre Beziehungen zu Anpassungserscheinungen direkt als nnantastbar feststehend hingestellt. 
allmählich die Form des normalen an. Im Unterkiefer erfolgte nach dem Abstoßen des Wundschorfs und der Epithelüberkleidung eine Abrundung und im weiteren Verlaufe eine Znspitzung der verletzten Stelle, während gleichzeitig das Wachstum vor sich ging. Die fertigen Regenerate haben in der Schuppenbekleidung und in der histologischen Beschaffenheit mancherlei Unterschiede von den entsprechenden Kieferteilen bei normalen Tieren aufzuweisen. Anstatt des ejnen Schildes, welches den amputierten Teil bedeckte (Fig. 1), sind bei manchen Exemplaren mehrere kleinere Schilder und bei andern sehr viele ganz kleine Schuppen (Granulaschuppen) am Regenerat zu sehen (Fig. 3 und 4). Betreffs der histologischen Beschaffenheit des Regenerats ist zu erwähnen, daß es mir bis nun nicht gelungen ist mit Sicherheit festzustellen, ob die Knochen der Kiefer als solche regeneriert werden können. Wie ich bereits früher schon mitgeteilt habe, habe ich an Querschnitten der regenerierten Kieferteile Knorpelgewebe anstatt des Knochengewebes konstatiert. Ich vermutete damals, daß dieses Knorpelgewebe mit zunehmendem Alter möglicherweise einer Ossification unterliege, was ich auch bei der Regeneration des Schwanzes bei Eidechsen voraussetzte. Nach einer abermaligen mikroskopisehen Untersuchung fand ich aber an Querdurchschnitten (Fig. 22 und 23) von andern Exemplaren kein Knorpelgewebe, sondern ein Bindegewebe mit sehr reichlich eingelagerten Kernen (Fig. 24). Ich glaube nun, daß der RegenerationsprozeB der Knochen hier in der Weise verläuft, daß ein Bindegewebe gebildet wird als Vorstufe des Knorpelgewebes, welch letzteres möglicherweise mit zunehmendem Alte des Regenerats einer Ossification unterliegt. Eine sichere Aufklärung über diesen interessanten Gegenstand könnte wohl eine eingehendere mikroskopische Untersuchung während des Regenerationsprozesses gewähren, wozu allerdings sehr viele Zwischenstadien serienweisse konserviert werden müBten.

An der Hand der diesmal angestellten Versuche an derselben Tierart unternahm ich es zu ermitteln, ob anch die weiteren Partien der Kiefer zu regenerieren vermögen. Zu diesem Zwecke habe ich bei der diesmaligen Operation den Schnitt tiefer geführt, so, daß auch die Nasenlöcher weggeschnitten wurden. Der Versuch wurde in zwei Serien aufgestellt. In der ersten Serie wurden 25 Exemplare am Oberkiefer, in der zweiten Serie 8 Exemplare an beiden Kiefern operiert. Die Blutung war eine sehr starke. Manche Tiere sind gleich nach der Operation eingegangen, bei einigen andern war die Blutung so stark, daß trotz der Hemmung die Mundöffnung sich mit 
Blut verklebt hat und die Tiere erstickt sind. Bei dem am Leben gebliebenen ging der Wundheilungsprozeß sebr rasch vor sich. Schon zwei Tage nach der Operation schlossen sich die Wundränder und kurz darauf konnte man wahrnehmen, daß die Wundliicke sich füllt und hervorwölbt. In dem noch ziemlich lockeren Gewebe des Verschlusses der Wundlücke konnte ich nach überraschend kurzer Zeit eine Öffnung und zwei Tage später eine zweite Öffnung an der Stelle, welche im normalen Zustande den Nasenlöchern zukommt, bemerken. Es ist sehr wahrscheinlich, daß dies in Ausbildung begriffene Nasenlöcher waren. Leider konnte ich diese Erscheinung nicht weiter verfolgen, da das letzte noch am Leben gebliebene Exemplar, bei dem ich das beobachtet habe, sehr bald einging. Durch die sehr starke Verletzung waren nämlich die Tiere an der Nahrungsaufnahme verhindert. Dies und auch die sehr geringe Widerstandsfähigkeit der Eidechsen gegen Verletzungen zur Winterszeit brachten die Tiere zum gänzlichen Absterben. Ich habe mich zwar bemuiht die Tiere am Leben zu erhalten, indem ich jedes einzelne Tier futterte, aber - war die auf diese Weise von den Tieren aufgenommene Nahrung unzureichend, oder war es der Einfluß der ungtinstigen Jahreszeit - es gelang mir nicht einmal ein einziges Exemplar am Leben zu erhalten. Die Tiere wurden, wie erwälnt, künstlich gefüttert, nämlich mit Larren vom Mehlkäfer (Tenebrio molitor) gestopft. Dies wurde in der Weise vollzogen, daB ich der Larve den Kopf abgeschnitten habe und sie dann dem Tiere vorhielt, welches den herausquellenden Körpersaft gierig leckte und dabei den Mund sehr weit öffnete, so, daß man nun - allerdings sehr vorsichtig, weil sonst das Tier ersticken könnte - das Futter mittels Pinzette hineinschieben konnte. Trotzdem dieser Versuch an der großen Sterblichkeit der Tiere scheiterte, scheint es mir doch nicht ansgeschlossen zu sein, daß die tiefer liegenden Partien der Kiefer zu regenerieren vermögen und es würde sich vielleicht verlohnen den Versuch noch einmal - selbstverständlich zu einer günstigeren Jahreszeit (Frühjahr und Sommer) und an einer größeren Anzahl von Versuchstieren - anzustellen.

Die Amputation des Oberkiefers bis zur Grenze der Nasenlöcher und eines entsprechend großen Teils am Unterkiefer führte ich anch bei andern Lacertiden: Lacerta vivipara Jacqu. und Lacerta viridis Laur. aus. Aber auch dieser Versuch ergab kein positives Resultat. Die Anzahl der Versuchstiere war im Verhältnis zur großen Sterblichkeit eine zu geringe. Auch scheint bei diesen Tieren die Wider- 
standsfähigkeit - wenigstens bei Verwundungen am vorderen Körperende - eine geringere zu sein, als bei der Lacerta agilis. Bei Lacerta vivipara verlief die Wundheilung ganz äbnlich wie bei Lacerta agitis und sicherlich wäre hier ein positives Resultat erzielt worden, wenn nicht die erwähnten ungünstigen Umstände mitgewirkt hätten. Die Tiere sind alle binnen etwa 20 Tagen eingegangen. Hingegen scheint es mir zweifelhaft, ob die Kiefer bei den Lacerta viridis regeneriert hätten, selbst wenn die Tiere längere Zeit am Leben geblieben wären. Zwar wirkten auch hier dieselben mißgünstigen Umstände, aber dennoch zeigten diese Tiere eine noch viel geringere Widerstandsfahigkeit, als die andern Lacertiden. Die Tiere sind nämlich alle nach etwa 8-10 Tagen eingegangen, ohne dals sich die Wunden geschlossen lätten, was möglicherweise auf eine Infektion zurückzufuihren ist.

\section{Tarentola annularis Geoffr. und mauretanica $L$.}

Auch zwei Vertreter der Geckoniden, nämlich Tarentola annularis und Tarentola mauretanica wurden zu diesen Versuchen herangezogen. Das Terrarium, in dem die Tiere untergebracht waren, war aus Holz, mit Glasscheiben versehen, der Boden war mit Sand ausgebettet und jedes Terrarium enthielt einen kleinen Baumast und einige Steine, unter welche sich die Tiere besonders bei trübem Wetter gern verkriechen und oft haufenweise neben- und aufeinander liegen. Bei schönem Wetter waren die Tiere immer am Baumast oder an den Wänden des Terrariums zu sehen. Der Sandboden erwies sich aber als sehr unpraktisch, weil an den Wunden immer Sandkörnchen blieben, was die Wundheilung verzögerte. Deshalb bettete ich dann den Boden mit frischem, aber trockenem Moos ans, das oft gewechselt wurde. Als Futter wurden auch hier Mehlkäferlarven verwendet.

Der Regenerationsprozeß verlief hier ganz ähnlich wie bei L. agilis. Es erfolgte also zunächst im Oberkiefer der Verschluß der Wundränder und fortschreitendes Aneinanderricken der Winkelarme der Wundlïcke, bis diese gänzlich schwand; am Unterkiefer erfolgte nach dem Wundverschluß eine Abrundung und fortschreitende Zuspitzung bei gleichzeitigem Nachwachsen desselben. Hierauf konnte man die langsame Differenzierung des Epithels und die Bildung der damit zusammenhängenden Schuppenbekleidung beobachten. Was diese letztere betrifft, sei darauf hingewiesen, daß sie gewisse - wenn auch nicht sehr wesentliche - Abweichungen von der primären 
Beschuppung insofern aufweist, als die Schilder des Regenerats ein wenig anders gestaltet sind als die an derselben stelle beim normalen Tiere (Fig. 7-14). Hier ist nämlich äbnlich wie bei L. agilis eine Zersplitterung des einen, die amputierte Stelle bedeckenden Schildes in zwei oder mehrere kleinere Schilder zu bemerken (Fig. 7, 8, 9, 10, 13,14) oder nimmt das Schild am Regenerat des Unterkiefers eine andre (flaschenförmige) Gestalt an. - Was die zur Regeneration erforderliche Zeitdauer betrifft, ist diese eine größere als bei $L$. agilis. Auch ist hier die Widerstandsfähigkeit eine geringere, da nämlich die Sterblichkeit nach Verletzungen ziemlich groß ist. Das Regenerat erreicht bei Tarentola annularis und mauretanica die normale Größe; Tiere mit regenerierten Kieferspitzen sind von normalen kaum zu unterscheiden.

Betreffs der Regeneration der Kiefer bei Amphibien existiert nur eine ältere Angabe 1) iber die Kieferregeneration bei den Tritonen. Ich unternahm es daher, die Regenerationsfähigkeit der Kiefer bei Triton cristatus und Triton alpestris nachzuprïfen, um zu sehen, ob auch hier, wie bei den Eidechsen, die histologische Beschaffenheit. des Regenerats eine andre ist als die der amputierten Kieferteile; ferner stellte ich dieselben Versuche bei den höher stehenden schwanzlosen Amphibien bei Tieren in versehiedenen Entwicklungsstadien an, um zu sehen, ob die phylogenetische und ontogenetische Entwicklungsstufe in bezug auf die Regeneration zum Ausdruck gelangt. Untersucht wurden: Triton cristatus, Thiton alpestris, Rana esculcnta, Rame temporaria und Hyla arborea.

\section{Triton cristatus Laur. und alpestris Laur.}

Bei Triton cristatus nod $T r$. alpestris amputierte ich am Oberkiefer die Spitze bis zur Grenze der Nasenlöcher und am Unterkiefer ein entsprechend großes Stiick. Die Blutung war in beiden Fällen eine äußerst geringe. Die Wundstellen iiberkleideten sich sebr rasch mit einer lichten Epithelschicht, die immer dunkler wurde, indem zuerst das schwarze und erst später das gelbe bzw. rötliche Pigment auftrat. Die Regeneration erfolgte sehr rasch; bei Tr. cristatus nach 6-8 Wochen, bei Tr. alpestris danerte der RegenerationsprozeB etwas

1) Spallanzanr, Prodromo di un opera imprimersi sopra le riproduzioni animali dato in luce dall' abate Spalidanzani. Modena 1768. 
länger, 10-12 Wochen. Das Regenerat (Fig. 15) ist in beiden Fällen ein vollständiges; sogar die Zähne werden neugebildet (Fig. 23). Der Querschnitt durch das Regenerat weist nicht den geringsten Unterschied von dem Querschnitt an derselben Stelle des Kiefers bei einem normalen Tiere auf. Auch im äußeren Habitus ist weder ein Triton alpestris noch ein Tr. cristatus mit Regenerat von einem normalen Tiere zu unterscheiden.

\section{Rana esculenta L.}

Hier stellte ich den Versuch an Kaulquappen und an ausgebildeten Tieren, deren Körpergröße etwa 2,3 cm betrug, an. An den Kaulquappen amputierte ich ringsherum den Hornschnabel weg und an den ausgebildeten Tieren am Oberkiefer die Spitze bis zu den Nasenlöchern und ein entsprechend großes Stück der Unterkieferspitze. Die Kaulquappen, welche in einer Anzahl von 26 Exemplaren operiert wurden, zeigten eine geringe Widerstandsfähigkeit gegen derartige Verletzungen und starben beinahe alle nach wenigen Wochen ab. Nur drei Exemplare sind am Leben geblieben und regenerierten den entfernten Teil des Vorderendes, nämlich zuerst den Oberkiefer und dann erst den Unterkiefer, vollständig. - Die ausgebildeten Frösche wurden in einer Anzahl von 22 Exemplaren operiert. Auch hier war die Widerstandsfähigkeit eine verhältnismäßig geringe. Die Blutnng war eine ganz unbedeutende. Die Tiere wurden mit Mehlkäferlarven gefüttert. Der Verlauf der Regeneration war hier ganz ähnlich wie bei Lacerta agilis und bei den Tritonen, und nahm einen Zeitraum von etwa 6-8 Wochen in Ansprnch. Die Regenerate (Fig. 5) sind vollständig; die amputierten Knochenstiicke sind nengebildet worden, wovon ich mich durch Maceration überzeugte. Ein Tier mit regenerierten Kieferspitzen ist von einem normalen gar nicht zu unterscheiden.

\section{Rana temporaria L.}

Der Versuch wurde an zehn Exemplaren verschiedener Größe vorgenommen, die jedoch alle eine Länge von über $4 \mathrm{~cm}$ anfwiesen. Hier amputierte ich nur die Spitze des Oberkiefers bis zu den Nasenlöchern, der Unterkiefer blieb intakt. Die Blutung war hier eine ziemlich starke und der $W$ undverschluß erfolgte bei den meisten Tieren erst nach 9 Tagen, bei andern noch später. Hierauf folgte an den Rändern der Wundlücke eine Zellwucherung, welche einen geringen 
Teil der Wundlïcke ausfüllte und eine zweizackige Form annahm. Am Unterkiefer hat die entsprechende Stelle hypertrophiert und eine zweizackenförmige Gestalt angenommen, so daß die Hebungen am Unterkiefer in die Senkungen am Oberkiefer (und umgekehrt) greifen (Fig. 5). Diese Erscheinung ist als kompensatorische Hypertrophie zu bezeichnen. Regeneriert hat in einem Zeitraum von 6 Monaten kein einziges Tier, was meiner Ansicht nach darauf zurückzuführe ist, daß die Tiere schon ziemlich groß waren, da die Operation vorgenommen wurde.

\section{Hyla arborea $L$.}

Hier stellte ich den Versuch in zwei Serien auf. Als erste Serie sind zehn Exemplare von einer Größe von $2,5 \mathrm{~cm}$ operiert worden. Amputiert wurde nur die Oberkieferspitze bis zu den Nasenlöchern. Die Blutung war eine geringe. Der Regenerationsprozeß verlief genau so wie bei Rana csculenta; nur nahm die Regeneration einen etwas gröBeren Zeitraum in Anspruch. Nach etwa 31/2 Monaten konstatierte ich vollständige Regenerate des verletzten Oberkiefers bei vier Exemplaren. Das Regenerat (Fig. 17, 19) zeigt geringe Unterschiede gegenüber derselben Stelle am normalen Tiere (Fig. 16, 18), welche auf der Unterseite des Oberkiefers zu konstatieren sind. Namentlich ist am Regenerat eine leichte Einsenkung der Kieferspitze und eine viel kleinere Entfernung des in der Mitte verlaufenden Bogens von den Nasenlöchern, wie auch eine Verdickung und sehr lichte Färbung des Bogens zu bemerken. Als zweite Serie wurden acht Exemplare in der Größe von $5 \mathrm{~cm}$ operiert. Diese Tiere regenerierten aber (wahrscheinlich wegen vorgeschrittenen Alters) nicht mehr. Der Versuch wurde nach 6 Monaten mit negativen Resultaten abgeschlossen. -

Die Resultate obiger Versuche lassen sich dahin zusammenfassen:

I. Von den Amphibien regenerieren: a) die Urodelen (Triton cristatus und alpestris) die amputierten Kieferspitzen gänzlich. Das Alter der Tiere spielt hier keine Rolle; b) bei den Anuren nur Kaulquappen und kleinere Tiere (Rana esculenta, Hyla arborea), während bei größeren Tieren die amputierten Kiefer gar nicht regenerieren (Hyla arborea) oder eine Regulation durch kompensatorische Hypertrophie eintritt, wenn blob ein Kiefer amputiert wurde (Rana temporaria). 
II. Von den Reptilien regenerierten amputierte Kieferspitzen bis zur Grenze der Nasenlöcher: die Eidechse Lacerta agilis, die Geckone Tarentola annularis und mauretanica. Das Regenerat weist eine von der primären abweichende Beschuppung auf.

III. a) bei den Amphibien werden im amputierten Kieferteile alle Gewebsarten vollständig regeneriert, insofern das betreffende Tier die Regenerationsfähigkeit des Kiefers noch besitzt (Tritone, Rana esculenta, Hyla arborea); b) bei den Reptilien konnte nicht beobachtet werden, daß das Knochengewebe in den amputierten Kieferteilen regeneriert werde. Die Regenerate zeigen bei den Reptilien an Stelle des Knochengewebes im amputierten Kieferteile ein Ersatzgewebe (Bindegewebe oder in günstigsten Fiallen Knorpelgewebe).

IV. Die Regenerationsfähigkeit der Kieferspitzen bei den untersuchten Amphibien und Reptilien nimmt a) mit der höheren phylogenetischen Stellung (Tritone, Frösche, Echsen) und b) mit der höheren ontogenetischen Entwicklungsstufe des Individuums (Kaulquappen, kleine Tiere, ausgewachsene Tiere) stufenweise ab.

\section{Versuchsprotokolle.}

Lacerta agilis (I. Serie).

\begin{tabular}{|c|c|c|c|c|c|c|c|}
\hline $\begin{array}{c}\text { Art der Operation } \\
\text { und } Z \text { ustand der operierten } \\
\text { Tiere }\end{array}$ & $\begin{array}{l}\text { An- } \\
\text { zahl }\end{array}$ & $\begin{array}{c}\text { Tag der } \\
\text { Operation }\end{array}$ & Kontrolliert & $\begin{array}{l}\text { Zu- } \\
\text { grande } \\
\text { ge- } \\
\text { gangen }\end{array}$ & $\mid \begin{array}{c}\text { Nicht } \\
\text { re- } \\
\text { gene- } \\
\text { riert }\end{array}$ & $\begin{array}{l}\text { Re- } \\
\text { gene- } \\
\text { riert }\end{array}$ & $\begin{array}{l}\text { Regenerier- } \\
\text { ter 'Teil }\end{array}$ \\
\hline $\begin{array}{l}\text { Oberkieferspitze mit den } \\
\text { Nasenlochern amputiert }\end{array}$ & 25 & 31. X. 04 & & & & & \\
\hline $\begin{array}{l}\text { Erstickt durch Verklebung } \\
\text { der Mundöffuung mit } \\
\text { Blnt......... }\end{array}$ & & & 1. XI. 04 & 2 & & & \\
\hline Wundverschlu $B . .$. & & & 3. XI. 04 & 7 & & & \\
\hline $\begin{array}{l}\text { Die Wundliicke füllt sich } \\
\text { und wölbt sich hervor. }\end{array}$ & & & 9. XI. 04 & 8 & & & \\
\hline $\begin{array}{l}\text { Bildung eines Nasenloches } \\
\text { in der Wölbung der ver- } \\
\text { wachsenen Wundlicke } \\
\text { bei einem Exemplar? }\end{array}$ & & & 13. XI. 04 & 4 & & & \\
\hline $\begin{array}{l}\text { Bildung des zweiten } \mathrm{Na}= \\
\text { senloches bei demselben } \\
\text { Exemplar? . . . . . }\end{array}$ & & & 15. XI. 04 & 2 & & & \\
\hline $\begin{array}{l}\text { Die letzten Exemplare ab- } \\
\text { gestorben. . . . . . }\end{array}$ & & & 21. XI. 04 & 2 & & 0 & \\
\hline
\end{tabular}


Lacerta agilis (II. Serie).

\begin{tabular}{|c|c|c|c|c|c|c|c|}
\hline $\begin{array}{c}\text { Art der Operation } \\
\text { und Zustand der operierten } \\
\text { Tiere }\end{array}$ & $\begin{array}{l}\text { An- } \\
\text { zahl }\end{array}$ & $\begin{array}{c}\text { Tag der } \\
\text { Operation }\end{array}$ & Kontrolliert & $\begin{array}{c}\mathrm{Zu}- \\
\text { grunde } \\
\text { ge- } \\
\text { gangen }\end{array}$ & $\left\{\begin{array}{c}\text { Nicht } \\
\text { re- } \\
\text { gene- } \\
\text { riert }\end{array}\right.$ & $\begin{array}{l}\text { Re- } \\
\text { gene- } \\
\text { riert }\end{array}$ & $\begin{array}{c}\text { Regenerier } \\
\text { ter Teil }\end{array}$ \\
\hline $\begin{array}{l}\text { Oberkiefer mit Nasen- } \\
\text { löchern amputiert; Un- } \\
\text { terkiefer entsprech. tief }\end{array}$ & 8 & 16. XII.04 & & & & & \\
\hline $\begin{array}{l}\text { Wundverschluß bei drei } \\
\text { Exemplaren ..... }\end{array}$ & & & 18. XII. 04 & 2 & & & \\
\hline $\begin{array}{c}\text { Wundverschlu } \beta \text { bei zwei } \\
\text { Exemplaren. . . . }\end{array}$ & & & 20. XII.04 & 2 & & & \\
\hline Die Wundlitcke füllt sich & & & 21. XII.04 & $\begin{array}{l}1 \\
2 \\
1\end{array}$ & & & \\
\hline Das letzte Exemplar tot & & & 29. XII. 04 & 1 & & 0 & \\
\hline
\end{tabular}

Tarentola annuleris.

Beide Kieferspitzen $\{0$ berkiefer bis zu den Nasenlöehern, Unterkiefer entsprech. tief) amputiert.

Wundverschlu6 . . . .

Wundlïcke im oberkiefer verkleinert, Unterkiefer abgerundet, die Wundsleidet . . . . . .

Regenerate (noch nicht vollständig). . . . .

Vollständige Regenerate mit abweichender Beschuppung . . . . . rïnder mit Epithel über-

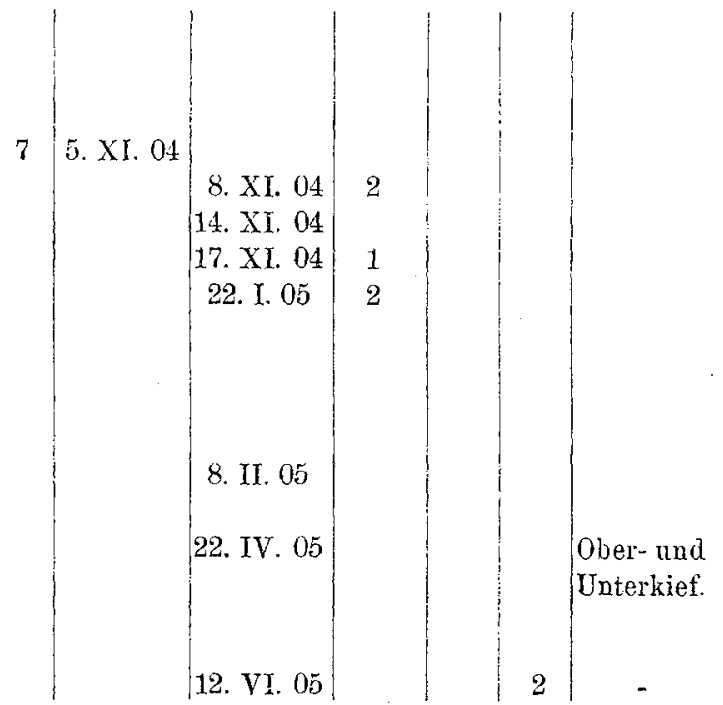

Tarentola mauretanica.

Beide Kieferspitzen (Oberkiefer bis zu den Nasenlöchern, Unterkiefer entsprech. tief) amputiert . Wundverschlu $3 . .$. .

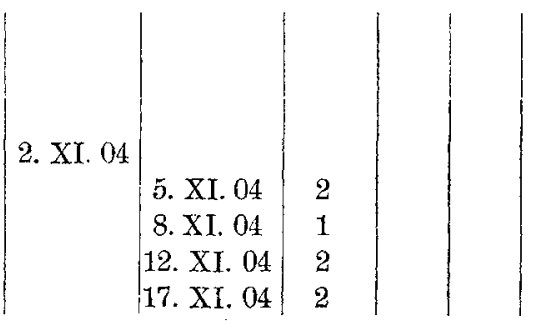




\begin{tabular}{|c|c|c|c|c|c|c|c|}
\hline $\begin{array}{c}\text { Art der Operation } \\
\text { und Zustand der operierten } \\
\text { Tiere }\end{array}$ & $\begin{array}{c}\text { An- } \\
\text { zahl }\end{array}$ & $\begin{array}{l}\text { Tag der } \\
\text { Operation }\end{array}$ & Kontrolliert & $\begin{array}{c}\text { Zu- } \\
\text { grunde } \\
\text { ge- } \\
\text { gangen }\end{array}$ & $\begin{array}{c}\text { Nicht } \\
\text { re- } \\
\text { gene- } \\
\text { riert }\end{array}$ & $\begin{array}{c}\text { Re- } \\
\text { gene- } \\
\text { riert }\end{array}$ & $\begin{array}{l}\text { Regenerier- } \\
\text { ter 'Teil }\end{array}$ \\
\hline $\begin{array}{l}\text { Wundluicke im Oberkiefer } \\
\text { verkleinert, Unterkiefer } \\
\text { abgerundet, die Wund- } \\
\text { ränder mit Epithel über- } \\
\text { kleidet. . . . . . } \\
\text { Regenerate (noch nicht } \\
\text { vollständig). . . . } \\
\text { Vollständige Regenerate } \\
\text { mit abweichender Be- } \\
\text { schuppung . . . . . }\end{array}$ & & & $\begin{array}{l}\text { 14. II. } 05 \\
22 . \text { IV. } 05\end{array}$ & 1 & & 3 & $\begin{array}{c}\text { Ober- и. } \\
\text { Unterkie- } \\
\text { ferspitze } \\
\text { - }\end{array}$ \\
\hline
\end{tabular}

Oberkiefer bis zu den $\mathrm{Na}-1$ senlöchern, Unterkiefer entsprechend tief amprtiert . . . . . . .

Wundverschluß u. Epithelbildung ......

Im Oberkiefer ist die Wundliicke beinahe ganz ansgefïllt, der Unterkiefer ist bedeutend nachgewachsen ....

Vollstaindige Regenerate.

Triton cristatus.

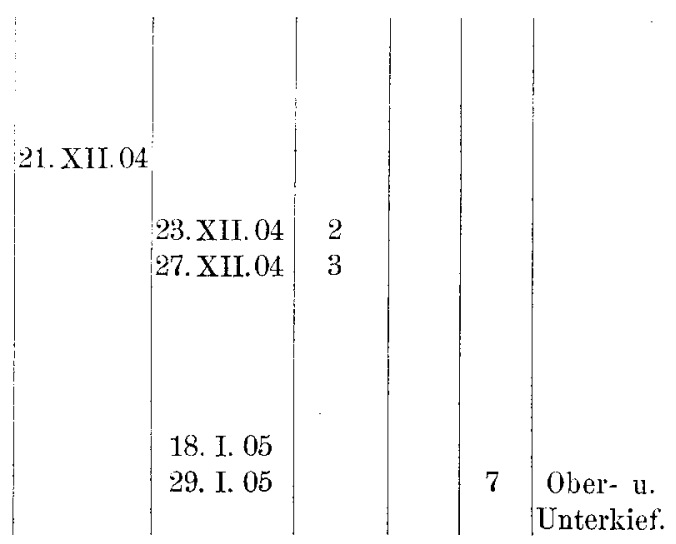

Triton alpestris.

Oberkiefer bis zn den Nasenlöchern, Unterkiefer entsprechend tief amputiert . . . . . . .

Wundverschluß 3 u. Epithelbildung. . . . . . .

Unterkiefer bedeut. nachgewachsen; im Oberkiefer ist die Wundlïcke nunmehr ganz klein. . Vollständige Regenerate.

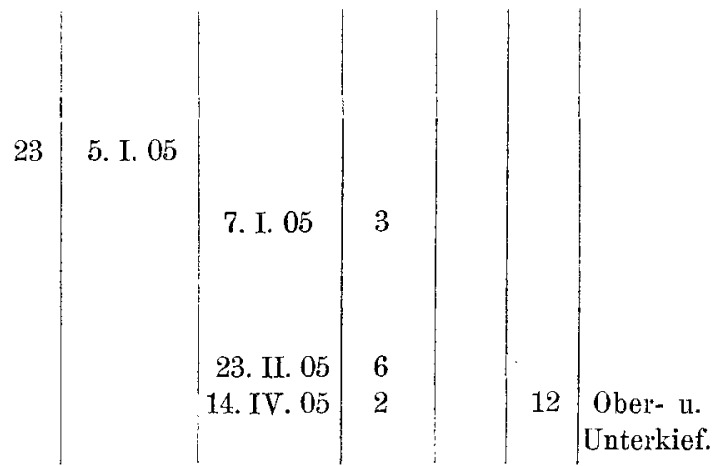


Rana esculenta (Kaulquappen).

\begin{tabular}{|c|c|c|c|c|c|c|c|}
\hline $\begin{array}{c}\text { Art der Operation } \\
\text { und Zustand der operierten } \\
\text { Tiere }\end{array}$ & $\begin{array}{c}\text { An- } \\
\text { zahl }\end{array}$ & $\begin{array}{c}\text { Tag der } \\
\text { operation }\end{array}$ & Kontrolliext & $\begin{array}{c}\text { Zn- } \\
\text { grunde } \\
\text { ge- } \\
\text { gangen }\end{array}$ & $\begin{array}{c}\text { Nicht } \\
\text { re- } \\
\text { gene- } \\
\text { riert }\end{array}$ & $\begin{array}{l}\text { Re- } \\
\text { gene- } \\
\text { jiert }\end{array}$ & $\begin{array}{l}\text { Regeneriex- } \\
\text { ter Teil }\end{array}$ \\
\hline $\begin{array}{l}\text { Entfernung des Horn- } \\
\text { schnabels mittels Schere }\end{array}$ & 26 & 4. I. 05 & & 7 & & 0 & \\
\hline $\begin{array}{c}\text { Wundverschluß u. Epithel- } \\
\text { bildung. . . . . . } \\
\text { - }\end{array}$ & & & $\begin{array}{c}\text { 7. I. } 05 \\
\text { 23. II. } 05\end{array}$ & $\begin{array}{l}5 \\
8\end{array}$ & & $\begin{array}{l}0 \\
0\end{array}$ & \\
\hline Regeneriert. . . . & & & 7. III. 05 & 0 & 2 & 4 & Oberkief. \\
\hline . . . & & & 18. III. 05 & 0 & 1 & 1 & - \\
\hline . . . . . & & & 27. III. 05 & 3 & 0 & 1 & - \\
\hline . . . . & & & 7. IV. 05 & 2 & 0 & 1 & $\begin{array}{l}\text { beide } \\
\text { Kiefer }\end{array}$ \\
\hline
\end{tabular}

Rance esculenta.

Entfernung beider Kieferspitzen...... . 22

Wundverschln $B$ u. Epithelbildung. . . . . . . Regenerat in Bildung. .

Vollständig regeneriert .

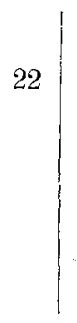

4. I. 05

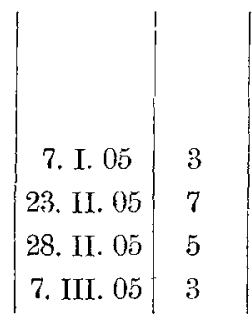

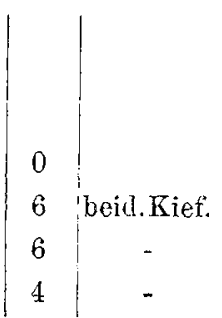

Rana temporaria.

Oberkieferspitze bis zu den Nasenlöchern heransgeschnitten ......

Wunde noch offen ...

Wundverschlu $\beta$. . . .

Zellwucherung an den Rändern der Wundlücke

Der der entfernten Oberkieferspitze entsprech. Teil am Unterkiefer ist hypertrophiert . . . .
6. I. 05

8. I. 0 อ̃

12. I. 05

17. I. 05

3. II. 05

14. II. 05

4. III. 05

21. IV. 05 2

abgeschlossen: 7. VI. 05

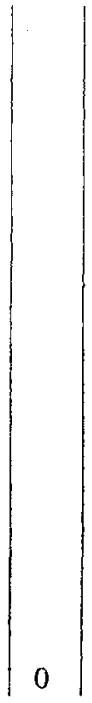


Hyla arborea [I. Serie (klein)].

\begin{tabular}{|c|c|c|c|c|c|c|c|}
\hline $\begin{array}{c}\text { Art der Operation } \\
\text { und Zustand der operierten } \\
\text { Tiere }\end{array}$ & $\begin{array}{l}\text { An- } \\
\text { zahl }\end{array}$ & $\begin{array}{c}\text { Tag der } \\
\text { Operation }\end{array}$ & Kontrolliert & $\begin{array}{c}\mathrm{Zu-} \\
\text { grunde } \\
\text { ge- } \\
\text { gangen }\end{array}$ & $\begin{array}{l}\text { Nicht } \\
\text { ro- } \\
\text { gene- } \\
\text { riert }\end{array}$ & $\begin{array}{l}\text { Re- } \\
\text { gene- } \\
\text { riert }\end{array}$ & $\begin{array}{l}\text { Regenerier- } \\
\text { ter Teil }\end{array}$ \\
\hline $\begin{array}{l}\text { Oberkieferspitze bis zu den } \\
\text { Nasenlöchern herausge- } \\
\text { schnitten....... }\end{array}$ & 10 & 7. I. 05 & & & & & \\
\hline $\begin{array}{cc}\text { Wundverschluß . . . } \\
- & .\end{array}$ & & & $\begin{array}{l}\text { 9. I. } 05 \\
\text { 13. I. } 05\end{array}$ & $\begin{array}{l}2 \\
4\end{array}$ & & & \\
\hline $\begin{array}{l}\text { Wundlïcke füllt sich; Re- } \\
\text { generat in Bildung }\end{array}$ & & & 29. I. 05 & 1 & & & \\
\hline Regenerate. . . . & & & 24. III. 05 & & & 3 & $\begin{array}{c}\text { Oberkief.- } \\
\text { spitze }\end{array}$ \\
\hline
\end{tabular}

Hyla arborea [II. Serie (groß)].

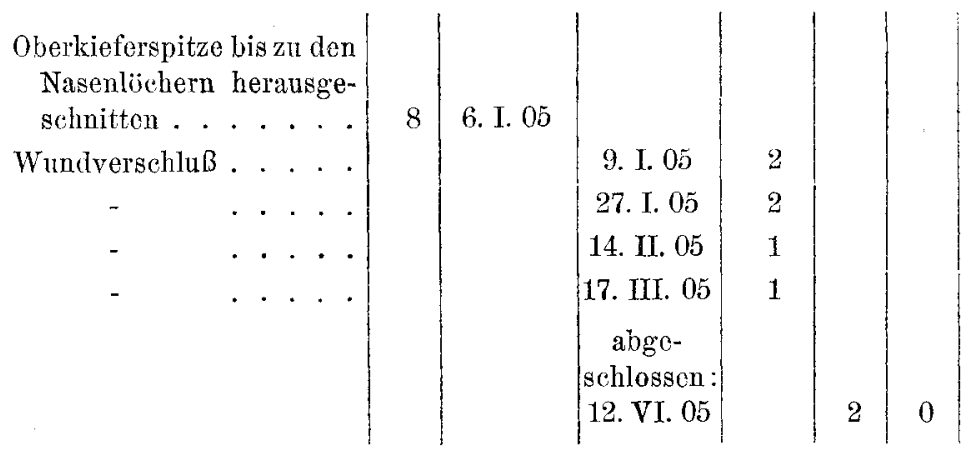

\section{Erklärung der Abbildungen.}

Tafel I.

(Sämtliche Figuren nat. Gr.)

Fig. 1. Kopf von Lacerta agitis mit normalen Kiefern (von unten gesehen).

Fig. 2. Kopf von Lacerta agilis mit in der Regeneration begriffenem Ober- und Unterkiefer (von unten gesehen).

Fig. 3. Kopf von Lacerta agilis mit vollstiindig regeneriertem Ober- und Unterkiefer (von unten gesehen).

Fig. 4. Kopf von Lacerta agilis mit regeneriertem Ober- und Unterkiefer (von unten gesehen).

Fig. 5. Rana esculenta mit regenerierten Kiefern.

Fig. 6. Rana temporaria mit verletztem Oberkiefer und kompensatorisch hypertrophiertem Unterkiefer.

Fig. 7. Tarentola mauretanica, Unterkiefer von unten, normal.

Fig. 8. Tarentola mauretanica, Unterkiefer von unten, regeneriert. 
14 Isaak Werber, Regeneration der Kiefer bei Reptilien und Amphibien.

Fig. 9. Tarentola mauretanica, Oberkiefer von oben, nolmal.

Fig. 10. Tarentola mauretanica, Oberkiefer von oben, regeneriert.

Fig. 11. Tarentola annularis, Unterkiefer von unten, normal.

Fig. 12. Tarentola annularis, Unterkiefer von unten, regeneriert.

Fig. 13. Tarentola annularis, oberkiefer von oben, normal.

Fig. 14. Tarentola anmularis, Oberkiefer von oben, regeneriert.

Fig. 15. Triton cristatus, Ober- und Unterkiefer von unten, regeneriert.

Fig. 16. Hyla arborea, Oberkiefer von oben, normal.

Fig. 17. Hyla arborea, Oberkiefer von oben, regeneriert.

Fig. 18. Hyla arborea, Oberkiefer von unten, normal.

Fig. 19. Hyla arborea, Oberkiefer von unten, regeneriert.

\section{Tafel II.}

(Fig. 20, 21 und 23 vergr. Obj. a* Oc. 4, Zxiss, Tischhöhe, Fig. 22 vergr. $0 b j .7$ Oc. 4, ZeIss.)

Fig. 20. Querschnitt durch die Oberkieferspitze von Lacerta agilis, normal.

Fig. 21. Querschnitt durch eine regenerierte Oberkieferspitze von Lacerta agilis.

(Die Schrumpfungen dürften durch die Präparation entstanden sein.)

Fig. 22. Ein Stück aus dem Bindegewebe des regenerierten Oberkiefers von Lacerta cogilis.

Fig. 23. Querschnitt durch das Regenerat der Oberkieferspitze von Triton cristatus. 


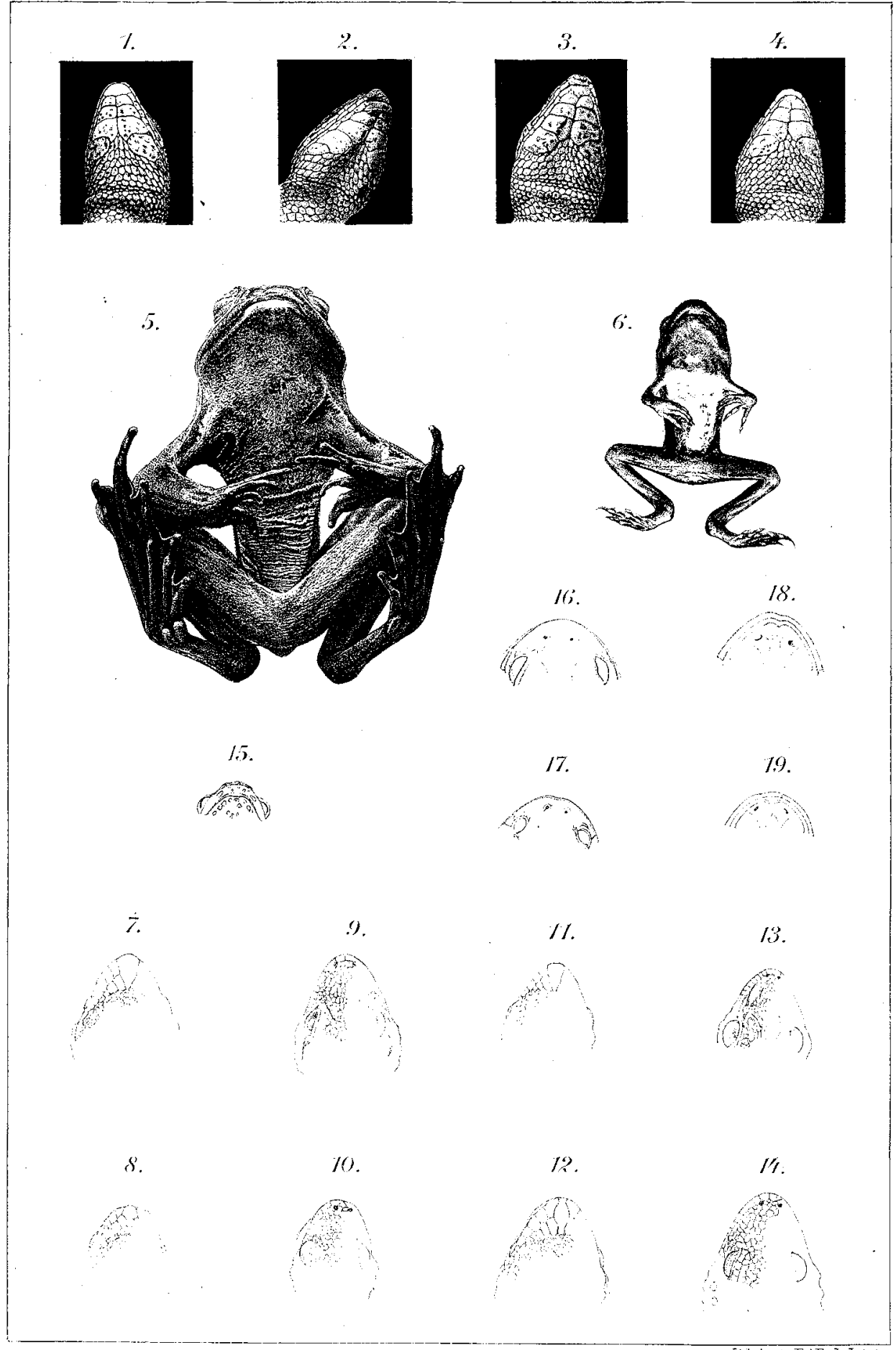




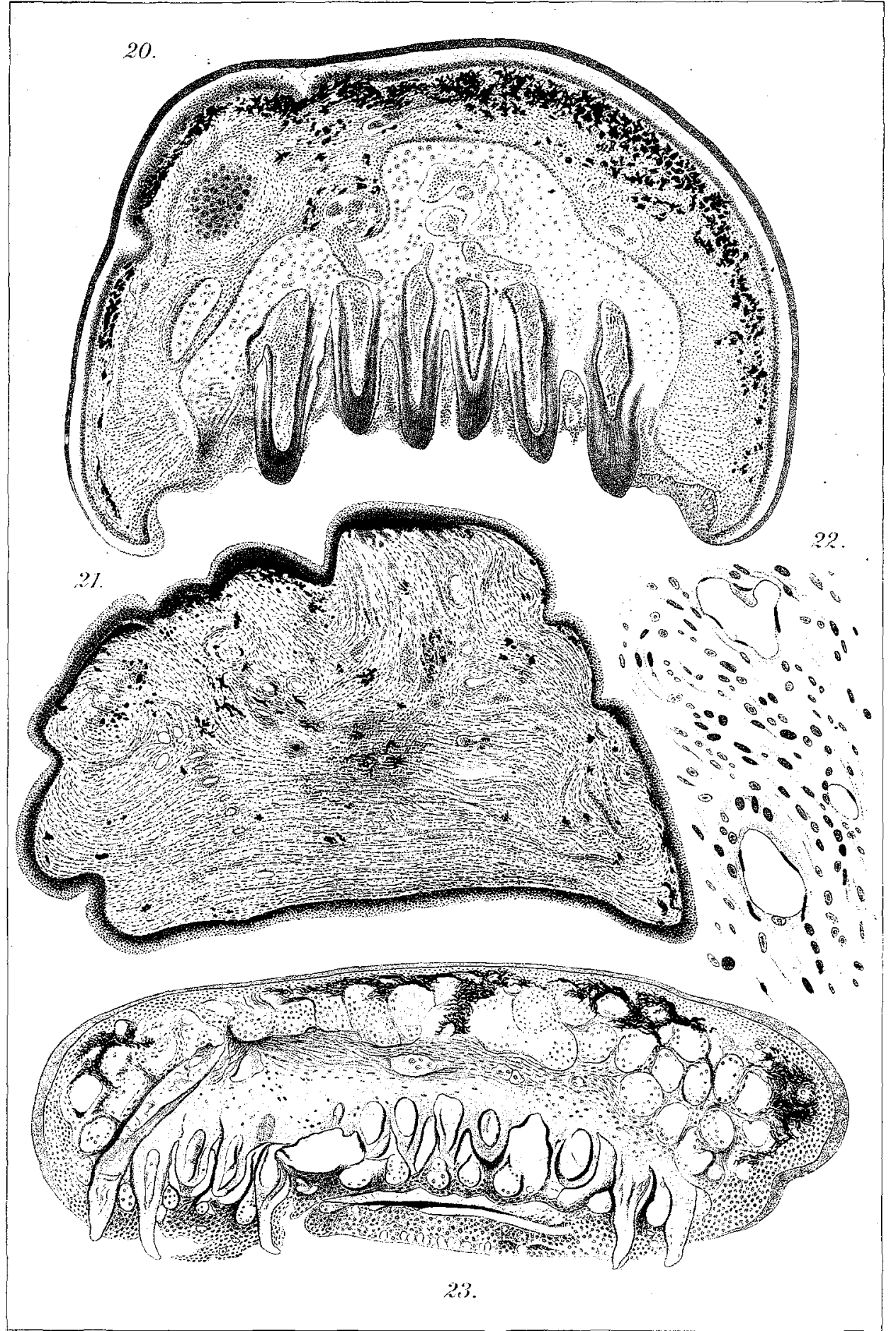

